\title{
J-value assessment of how best to combat COVID-19
}

\author{
Philip Thomas* \\ Safety Systems Research Centre, South West Nuclear Hub, Faculty of Engineering, University \\ of Bristol, Queen's Building, University Walk, Bristol BS8 1TR, UK
}

The new coronavirus infection will continue to pose a very severe challenge to the UK and to all countries around the world for the next 12 to 18 months. An epidemic model has been developed to explore the range of possible actions open to the UK and other nations to combat the virus. A "business as usual" policy would lead to the epidemic being over by September 2020, but such an approach would lead to a loss of life in the UK little less than what it suffered in the Second World War. Using the J-value without constraint suggests that exceptionally high spending would be justified for the three strategies that could reduce significantly the numbers of cases and deaths compared with the unmitigated epidemic. However such high spending is likely to come up against the J-value GDP constraint, whereby the measure should not so decrease GDP per head that the national population loses more life as a result of the countermeasure than it gains. The challenge for the UK Government (and other governments around the world) will be to manage its interventions so that the recession that is now inevitable is not significantly worse than that following the 2007-9 financial crash.

Keywords: coronavirus, COVID-19, economic challenge, epidemic model, J-value, lockdown

\section{Introduction}

Coronaviruses come from a large family of RNA viruses that cause respiratory infections, ranging from the common cold to more serious diseases. The Chinese authorities reported to the World Heath Organization (WHO) on 31 December 2019 that they had discovered a disease caused by a coronavirus that had not been seen before in humans. The source of the virus is believed to have been a food market in Wuhan City, where many animals of different species are kept together before being slaughtered for customers for food. It is thought that the virus jumped species there. The WHO has called the virus SARS-CoV-2, and named the illness it causes COVID-19— coronavirus disease 2019.

* E-mail: philip.thomas@bristol.ac.uk; research website: www.jvalue.co.uk 
The novelty of the virus in humans means that no immunity has built up against it. In $80 \%$ of cases the disease is relatively mild, but the other sufferers may experience a more severe infection, particularly those aged over 70 or with a preexisting medical condition. As of 22 March 2020, 335,511 cases had been reported worldwide, of whom 14,611 had died; 97,636 were known to have recovered. There were 5638 known cases in the UK at the same date, of whom 281 had died. ${ }^{1}$

Strong restrictions have been imposed on their citizens by many countries, including the UK, so as to reduce contact between people in an effort to lower the number of infections an infectious person passes on. These measures have had the effect of dampening economic activity severely.

This paper will use the Judgment- or J-value ${ }^{2}$ framework to explore the question of how much it is reasonable to spend on countermeasures. To do this, it is necessary to estimate the beneficial consequences in terms of prolongation of life that the countermeasure brings about. There is thus a requirement to model the dynamics of the spread of the epidemic based on available data.

A succinct statement of the utility of a mathematical model is provided by Ludwik Finkelstein: ${ }^{3}$

The essential value of models is that operations on the models enable conclusions to be drawn about the behaviour of the system represented.

Adding this to Sir Karl Popper's view ${ }^{4}$ that

the following maxim holds for all sciences: Never aim for more precision than

is required for the problem in hand.

and it may be concluded that simplicity should be a major aim for a useful model. A straightforward model is particularly appropriate when, as now, there are limitations in the data that impede the building of a more complex version. Thus, a simple mathematical description will be derived for the COVID-19 epidemic. The model is based on an adaptation of a point model of the dynamics of a nuclear reactor, ${ }^{5}$ which has structural similarities in the mathematics. Algebraic rearrangement of the final model shows that the coupled differential equations produced are equivalent to the SIR model (susceptible-infected-recovered) ${ }^{6}$ often used in epidemiological studies. The model's predictions will be compared against recorded data to this point in time and against those of the Imperial College model, which have been reported to Government.

1 Worldometer, COVID-19 Coronavirus Pandemic, updated 22 March $2020 \mathrm{https}: / /$ www.worldometers.info/coronavirus/

2 Thomas, P. J., Stupples, D. W. and Alghaffar, M. A., The extent of regulatory consensus on health and safety expenditure. Part 1: Development of the J-value technique and evaluation of regulators' recommendations. Process Safety and Environmental Protection 84(B5) (2006) 329-336.

3 Finkelstein, L., From technology to wider knowledge, understanding and wisdom. Measurement Control (Special Feature on Systems and Risk) 39 (2006) 268-272.

4 Popper, K. R., Realism and the Aim of Science (Preface, first published in 1956). London: Hutchinson (1983).

5 Thomas, P., Simulation of Industrial Processes for Control Engineers, ch. 21 (Nuclear reactors). Oxford: Butterworth-Heinemann (1999).

6 Kermack, W. O. and McKendrick, A. G., A contribution to the mathematical theory of epidemics. Proc. R. Soc. Lond. A 115 (1927) 700-721. 
The layout of the paper is as follows. Section 2 will explain the J-value and use it to derive the worth of a year of life to the average UK citizen, and will then introduce a constraint for the case where the permissible level of spending is so great that it will adversely affect the nation's gross domestic product (GDP). Section 3 will present the data available to populate the epidemic model. The model makes the assumption that a person who has recovered from the disease will then be immune to it (there have been a few cases reported from Wuhan of people falling ill a second time but these probably occurred when a false negative occurred on their viral test ${ }^{7}$ ). A comparison will then be made between the model's predicted cumulative totals of infected people in the UK and the observed figure from 31 January to 22 March 2020. Section 4 will compare the model outputs with the results of the Imperial College epidemic model. Section 5 will discuss the five basic strategies available to the UK (and other developed countries) at this time, ranging from "business as usual" (Option 0) to a lockdown of 12 months in the hope of developing a vaccine and immunizing a substantial fraction of the UK population before the restrictions are lifted (Option 5). The benefits of the four active policy options will be established in terms of the reduction in life years lost compared with Option 0. Section 6.1 will apply the J-value in a straightforward way to evaluate the justifiable cost for each countermeasure. Section 6.2 will derive the numerical value of the maximum reduction in GDP permissible before the countermeasure causes greater loss of life than it averts. The difficult economic challenge facing the UK Government will be presented in numerical terms. Section 7 will discuss how far uncertainties in the data are likely to affect the main findings, and Section 8 presents the conclusions.

Appendix A derives the dynamic model of epidemic spread in an intuitively appealing way. The appendix includes a derivation of the fraction that will confer population ("herd") immunity. This epidemiological term is used to denote the smallest fraction of people (or animals, depending on context) who need to have gained immunity for an infection introduced into the population to be deprived of sufficient hosts to be sustained. The infection will then decline. Appendix B shows how the cumulative total of cases predicted by the model may be converted into loss of life expectancy, taking into account the variation in fatality rate with age. Appendix C explains how the value of a life-year may be found using the J-value.

\section{The J-value}

\subsection{The worth of a year of life in the UK}

The Judgment- or J-value allows an objective and validated assessment to be made of the maximum that ought to be spent on a measure to extend life expectancy. It is based on the life quality index, $Q$, devised by Nathwani, Lind and Pandey ${ }^{8}$ following a project discussion with Ernest Siddall, ${ }^{9}$ which may be written:

7 Zuo, M., Coronavirus: why do 'recovered' patients test positive again? South China Morning Post (4 March 2020) https://www.scmp.com/news/china/society/article/3065022/coronavirus-why-dorecovered-patients-test-positive-again

8 Nathwani, J.S., Lind, N.C. and Pandey, M.D., Affordable Safety by Choice: the Life Quality Method. University of Waterloo, Ontario: Institute for Risk Research (1997).

9 Lind, N. and Nathwani, J., Origin and development of the LQI. LQI Symposium in Kongens Lyngby, Denmark, 21-23 August 2012. 


$$
Q=G^{1-\varepsilon} X
$$

where $G$ is the average income, taken for ethical reasons to be annual GDP per person, $X$ is the population-average life expectancy and $\varepsilon$ is risk-aversion (or the negative of the elasticity of marginal utility of income). The same expression may be found from very general considerations using a method that parallels dimensional analysis. ${ }^{10}$

Perturbing equation (1) yields:

$$
\frac{\delta Q}{Q}=(1-\varepsilon) \frac{\delta G}{G}+\frac{\delta X}{X}
$$

where $\delta X$ is the average change in life expectancy across the population. The requirement that the safety measure should not produce a net disbenefit is that $\delta Q \geq 0$, with the limiting case occurring at $\delta Q=0$. Applying this condition to equation (2) gives the maximum it is worth paying per person protected per year to achieve a gain in population-average life expectancy, $\delta X$, as:

$$
-\delta G=\frac{G}{1-\varepsilon} \frac{\delta X}{X}
$$

where the negative sign preceding $\delta Q$ indicates a reduction in effective annual income.

The J-value is then the ratio of the actual cost per year, $\delta \hat{G}$, to the maximum reasonable cost per year:

$$
J=\frac{\delta \hat{G}}{\delta G}
$$

Hence the J-value may be written:

$$
J=\frac{1-\varepsilon}{G} \delta \hat{G} \frac{X}{\delta X} .
$$

$J>1$ implies that the safety measure is, in the absence of special pleading to the contrary, too expensive. At $J=1$, the amount per year actually being spent, $\delta \hat{G}$, will be equal to the maximum reasonable sum, $\delta G$, and

$$
\delta \hat{G}=\delta G=\frac{1}{1-\varepsilon} \frac{\delta X}{X} G .
$$

By assuming that all life-extending decisions are governed by a J-value of 1.0, Thomas and Waddington were able to validate this expression in 2017 as a descriptive model of the assessment process applied all over the world. ${ }^{11}$ Very good correspondence between actual and predicted population-average life expectancy against GDP per head (a correlation coefficient, $R$, of 0.77 ) was found for 180 out of the 193 nations recognized by the United Nations in 2009

\footnotetext{
${ }^{10}$ Thomas, P.J., Stupples, D.W. and Alghaffar, M.A., The extent of regulatory consensus on health and safety expenditure. Part 1: Development of the J-value technique and evaluation of regulators' recommendations. Process Safety and Environmental Protection 84(B5) (2006) 329-336.

11 Thomas, P. and Waddington, I., Validating the J-value safety assessment tool against pan-national data. Process Safety and Environmental Protection 112A (2017) 179-197.
} 
and excellent correspondence $(R=0.89)$ for 162 countries. In addition, the J-value provides the first causal explanation of the Preston curve of life expectancy at birth versus GDP per head. ${ }^{\mathbf{1 2}}$

The value of a life year (VOLY) is shown in Appendix A to take the form:

$$
\delta V^{(1)}=\frac{1-e^{-r^{*} X_{1}}}{r^{*}} \frac{1}{1-\varepsilon} \frac{1}{X_{1}} G
$$

where $X_{1}$ is the population-average life expectancy for the current generation and the bracketed superscript in $\delta V^{(1)}$ implies that this is for a single life year.

The value of risk-aversion for developed countries is found to be $\varepsilon=0.91$, while the social discount rate, $r^{*}$, should be taken ${ }^{11}$ as the rate of growth of GDP per head, found to be $2.03 \%$ per annum for the UK for the period 1961 to $2013 .{ }^{13}$ The UK GDP per head ${ }^{\mathbf{1 4}}$ in 2019 was $G=$ $£ 33,141$ while, from the latest ONS life tables (for 2016-8), $X_{1}=41.8$ years. ${ }^{15}$ Inserting these figures into equation (C.3) gives the best current estimate of the value of a life-year in the UK as:

$$
\delta V^{(1)}=£ 248,209 .
$$

This implies that the Government and commercial organizations in the UK would be justified in spending up to just under a quarter of a million pounds on a scheme that would extend by one year the life expectancy of a citizen in the group to be protected. ${ }^{\mathbf{1 6}}$

The approach applies the following steps. First, an estimate may be made using the model of the number of people dying at each age. This allows a calculation to be made of how many years of life expectancy will be lost to COVID-19 at each age and the total expected loss of life years may be found.

12 Preston, S.H., The changing relation between mortality and level of economic development. Population Studies 29 (1975) 231-248.

${ }^{13}$ World Bank, World Development Indicators: GDP per capita growth (annual \%), updated 1 February 2017. Available at http://data.worldbank.org/indicator/NY.GDP.PCAP.KD.ZG

${ }^{14}$ Office of National Statistics, Gross domestic product (average) per head, at current market prices: SA [seasonally adjusted] https://www.ons.gov.uk/economy/grossdomesticproductgdp/timeseries/ihxt/pn2

${ }^{15}$ Office of National Statistics, National Life Tables UK: 2016 to 2018 https://www.ons.gov.uk/ peoplepopulationandcommunity/birthsdeathsandmarriages/lifeexpectancies/bulletins/ nationallifetablesunitedkingdom/2016to2018

${ }^{16}$ The figure given in equation (7) differs markedly from the value assigned to the "quality-adjusted life year", QALY, used by the National Institute for Health and Care Excellence (NICE), which advises the National Health Service on whether a drug is to be regarded as cost-effective or not. NICE estimates that a year in full health is worth between $£ 20,000$ and $£ 30,000,{ }^{*}$ figures that have not increased since the Institute was set up in 1999. They bracket symmetrically the value of a life year, $£ 25,000$, that could be found by dividing the life expectancy of the average UK citizen of 1997 ( $\sim 40$ years) into the "value of a prevented fatality" (VPF), £1.0M, used by the Department for Transport (DfT) at that time. But it has been shown that the method used to find the VPF was invalid and that the monetary value assigned to the VPF is simply wrong and far too low. ${ }^{\dagger}$ NICE's value of the QALY is an even greater understatement since it takes account of neither inflation nor the growth of the economy during the last 23 years. * Sir Andrew Dillon (2015), "Carrying NICE over the threshold" https:// www.nice.org.uk/news/blog/carrying-nice-over-the-threshold ; $†$ Thomas, P. and Waddington, I., What is the value of life? A review of the value of a prevented fatality used by regulators and others in the UK. Nuclear Future 13 (2017) 32-39 (available at http://www.bristol.ac.uk/cabot/news/2017/ research-review-safety.html). 
Next, using the same process, the total number of life-years may be found that would be lost after implementation of the countermeasure. The reduction in life-years lost (or, equivalently, the restoration of life-years) is then multiplied by the value of a year of life given by equation (7) and the result is the maximum expenditure that should be contemplated for that measure.

\subsection{J-value GDP constraint for exceptionally large justifiable costs}

The derivation above of the value of a year of life rests on the normal economic assumption that the system is perturbed about its operating point and not moved to an entirely new region of operation. However, protection schemes should not be put in place if their costs are large enough to cause the nation's economic output to fall so significantly that that will cause more loss of life (than if the scheme had never been implemented).

In such circumstances a J-value GDP constraint needs to be applied, based on the causeand-effect relationship found by Thomas and Waddington in their validation of the J-value against pan-national data. ${ }^{11}$ As noted previously, that paper provides the first quantified causal explanation of the fact that we get to live longer as we grow richer in the way shown in the Preston curve. ${ }^{11}$ Additional work examining detailed statistics for the UK and 35 industrialized countries has further corroborated the J-value explanation for the phenomenon of greater national income per citizen causing their lives to be longer. ${ }^{17}$

In particular, the J-value approach reveals the following causal relationship between GDP per head $G$ (cause) and population-average life expectancy $X$ (effect):

$$
\frac{X}{X_{0}}=\left(\frac{G}{G_{0}}\right)^{1-\varepsilon}
$$

where $X_{0}$ is the population-average life expectancy and $G_{0}$ is GDP per head before the countermeasure is implemented. The situation may be clarified by expressing the fall in population-average life expectancy as $\Delta X=X_{0}-X$ and the fall in GDP per head as $\Delta G=G_{0}-G$, thus allowing equation ( 8 ) to be written:

$$
\frac{X_{0}-\Delta X}{X_{0}}=\left(\frac{G_{0}-\Delta G}{G_{0}}\right)^{1-\varepsilon} .
$$

If the countermeasure causes a fall in GDP per head, $\Delta G_{\text {with, }}$ so large that the drop in population-average life expectancy with the measure, $\Delta X_{\text {with }}$, exceeds the reduction in life expectancy without the countermeasure, $\Delta X_{\text {without }}$ :

$$
\Delta X_{\text {with }}>\Delta X_{\text {without }} \text {, }
$$

then the measure should not be adopted and other methods of mitigation should be sought. Condition (10) provides a J-value-based constraint to the results coming from applying the J-value in the normal way, as outlined in Section 2.1.

${ }^{17}$ Thomas, P., Corroboration of the J-value model for life-expectancy growth in industrialized countries. Nanotechnol. Perceptions 13 (2017) 31-44 (available at http://www.jvalue.co.uk/papers/ 2017-CorroborationOfJvalue.pdf ). 


\section{Data for the epidemic model}

The number of people in a susceptible population that will be infected by the average COVID-19 sufferer (the "basic reproduction number", $R_{0}$ ), was taken to be 2.35 , based on Wuhan data. ${ }^{18}$ The incubation period, $\tau_{\text {inc }}$, was taken to be 5 days based on the same study while the period of infectiousness was taken to be 10 days. ${ }^{19}$

The standard assumption is that a just-infected person will undergo an incubation period, $\tau_{\text {inc, }}$ and will then be liable to infect others for a risk period, $\tau_{\text {risk. }}$. It is possible, however, that the person may be infectious in the later stages of the incubation period. Allowing for this, a general statement of the average time, $\tau_{\text {inf }}$, to infect others, measured from the time the first person is infected, may be written as equation (A.1) of Appendix A:

$$
\tau_{\text {inf }}=r_{1} \tau_{\text {inc }}+r_{2} \tau_{\text {risk }}\left\{\begin{array}{l}
0<r_{1} \leq 1.0 \\
0<r_{2} \leq 1.0
\end{array}\right.
$$

When the person is not infectious during the incubation period, $r_{1}=1.0$ and when the infectiousness is uniformly distributed over the normal risk period, $r_{2}=0.5$.

These values for $r_{1}$ and $r_{2}$ were taken as the starting assumption, but after matching model predictions to recorded cases as of 19 March 2020, the coefficient $r_{1}$ was reduced slightly to 0.9 , implying that infectiousness begins 12 hours before symptoms show. Hence, the average time, $\tau_{\text {inf }}$, to infect others, from the moment of infection, was taken to be $0.9 \times 5+0.5 \times 10=9.5$ days.

Based on ONS figures, the UK population is taken to be 67 million.

Two people tested positive with the coronavirus SARS-CoV-2 in the UK at the end of January. Initializing the model with this number allows a comparison to be made with the recorded figures. Figure 1 shows the trend in recorded cases to 22 March 2020 to be captured reasonably accurately.

It is fortunate that little effect from the disease has been observed on children and young people. Moreover, in $80 \%$ of cases the symptoms are mild and the sufferer recovers after a week or so. However the incidence of serious effects rises with age, particularly after the age of 70 , when fatality rates begin to rise more sharply. Imperial College's COVID-19 Response Team suggested that the rate of death against age in the UK would follow Table $1 .{ }^{20}$ These rates, which are based on an analysis of data from Wuhan, assume that normal UK hospital capability is not overwhelmed, an important condition given the large numbers of cases that might occur.

The availability of ventilators and intensive care units in the UK is known be insufficient to deal with hundreds of thousands of cases. Therefore a number of scenarios are considered

${ }^{18}$ Kucharski, A. J., Russell, T.W., Diamond, C., Liu, Y., Edmunds, J., Funk, S. and Eggo, R. M., Early dynamics of transmission and control of Covid-19: a mathematical modelling study. Lancet (March 2020) https://doi.org/10.1016/S1473-3099(20)30144-4

${ }^{19}$ Anderson, R. M., Heesterbeek, H., Klinkenberg, D. and Hollingsworth, T. D., How will countrybased mitigation measures influence the course of the Covid-19 epidemic? Lancet (March 2020) https://doi.org/10.1016/S0140-6736(2)30567-5

${ }^{20}$ Ferguson, N. M. et al., Impact of non-pharmaceutical interventions (NPIs) to reduce Covid-19 mortality and healthcare demand (report). London: Imperial College (16 March 2020) https:// www.imperial.ac.uk/media/imperial-college/medicine/sph/ide/gida-fellowships/Imperial-CollegeCOVID19-NPI-modelling-16-03-2020.pdf 
where the more severe incidence of fatality reported in Wuhan ${ }^{21}$ applies. The Wuhan fatality rates are given in Table 2.

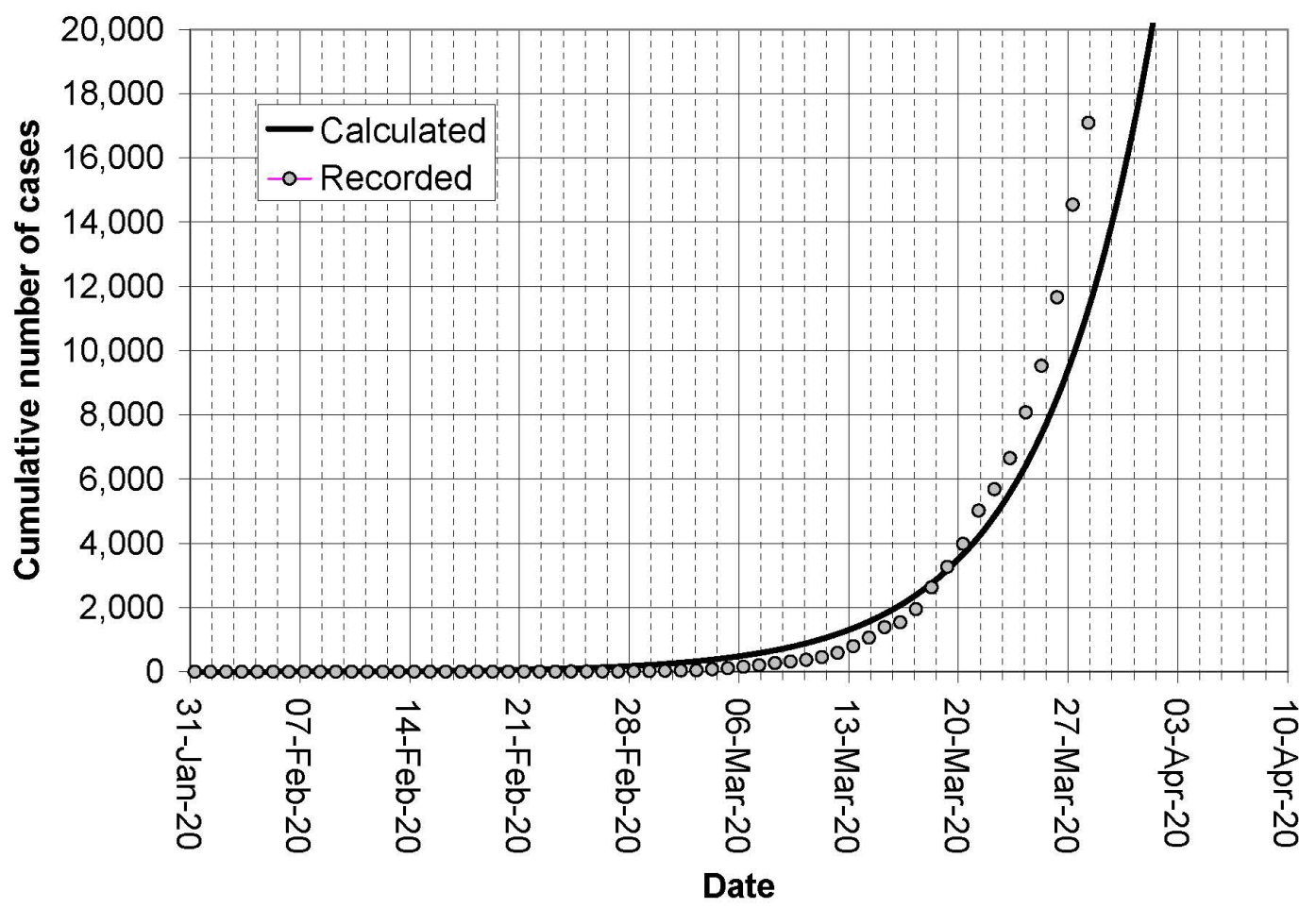

Figure 1. Comparison of this paper's model predictions with cumulative recorded cases

Table 1. Estimated age-specific fatality rates for the UK.

\begin{tabular}{cc}
\hline Age group (in years) & $\begin{array}{c}\text { Age-specific fatality } \\
\text { rate }\end{array}$ \\
\hline $0-9$ & $0.002 \%$ \\
$10-19$ & $0.006 \%$ \\
$20-29$ & $0.03 \%$ \\
$30-39$ & $0.08 \%$ \\
$40-49$ & $0.15 \%$ \\
$50-59$ & $0.60 \%$ \\
$60-69$ & $2.2 \%$ \\
$70-79$ & $5.1 \%$ \\
$80+$ & $9.3 \%$ \\
\hline
\end{tabular}

Table 2. Reported age-specific fatality rates for Wuhan.

\begin{tabular}{cc}
\hline Age group (in years) & $\begin{array}{c}\text { Age-specific fatality } \\
\text { rate }\end{array}$ \\
\hline $0-9$ & $0.0 \%$ \\
$10-19$ & $0.2 \%$ \\
$20-29$ & $0.2 \%$ \\
$30-39$ & $0.2 \%$ \\
$40-49$ & $0.4 \%$ \\
$50-59$ & $1.3 \%$ \\
$60-69$ & $3.6 \%$ \\
$70-79$ & $8.0 \%$ \\
$80+$ & $14.8 \%$ \\
\hline
\end{tabular}

${ }^{21}$ Worldometers, Age, Sex, Existing Conditions of Covid-19 Cases and Deaths https:// www.worldometers.info/coronavirus/coronavirus-age-sex-demographics/ 


\section{Comparison of the model given in this paper with that of Imperial College}

The Imperial College simulation for the "do nothing" ("business as usual") case assumed that the UK hospitals could cope with the numbers of coronavirus sufferers referred to them and so used the fatality rates of Table 1 .

Using the same figures in this model produces Figure 2, which shows the course of the epidemic in the absence of countermeasures in terms of the number of people suffering from COVID-19 as a function of time. The number of people suffering from the virus at any one time starts to rise very rapidly in the second half of April and reaches a peak of 14.2 million, just over $20 \%$ of the UK population, on 3 June 2020 . The number of people who have been infected at this point will be 38.5 million, which represents about $57.5 \%$ of the total population (in line with equation A.16). The number of sufferers will start to diminish from this point onwards, and the epidemic will be nearly over by the end of August. By that time, however, 58.4 million people would have been infected in total, representing $87 \%$ of the population.

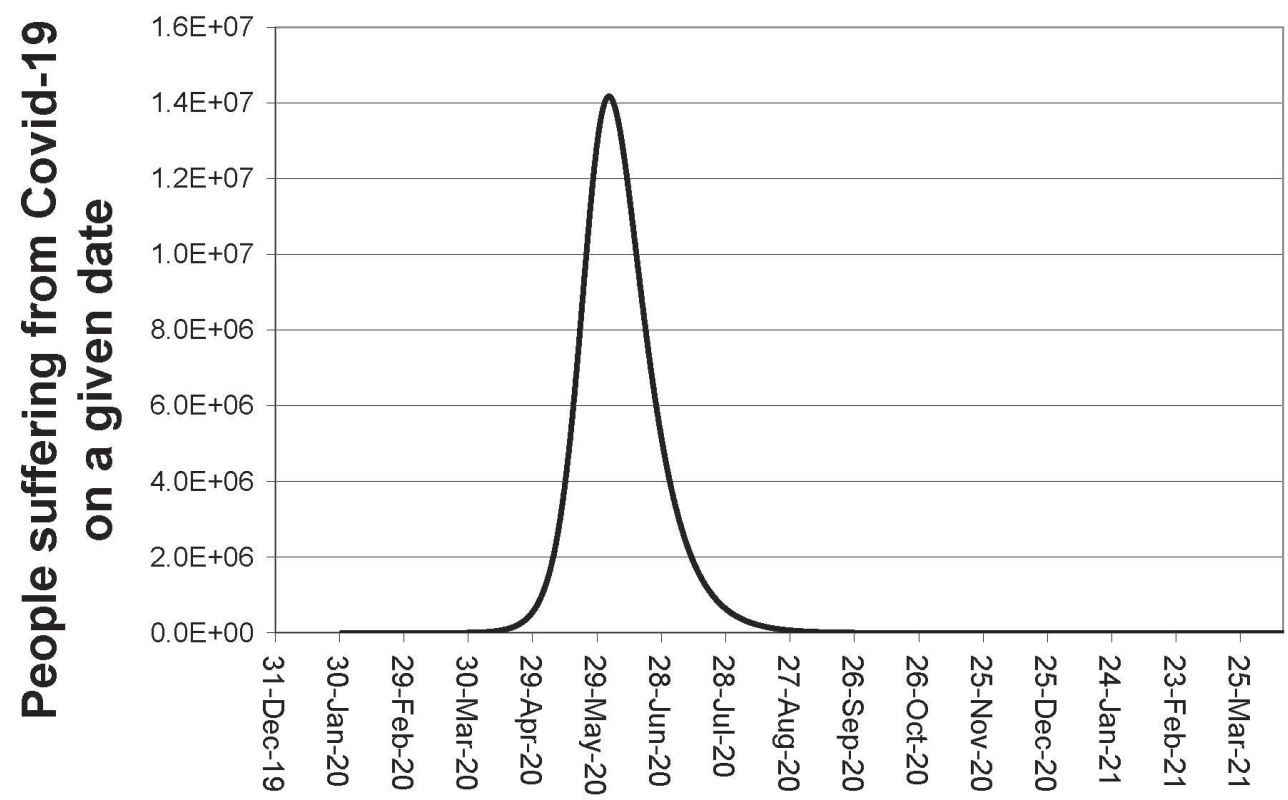

Date

Figure 2. Number of patients suffering from COVID-19 at a given date (Option 0).

The total number of deaths is calculated as 573,000 , which implies an overall death rate of $0.99 \%$. The amount of life expectancy lost is 8.4 million years and, on average, each victim loses 14.6 years of life.

For comparison, Ferguson et al., ${ }^{18}$ who set $R_{0}$ to 2.4 rather than the slightly lower figure of 2.35 used in this study, quote a cumulative total number of infections at the end of the epidemic of $81 \%$ of the UK population, implying 54.3 million people. They do not quote predicted numbers of people suffering from the virus each day, but their Figure 1 shows deaths peaking at 
around the middle of June, suggesting that the peak of infectious cases would have occurred around the end of May in their model (cf. 3 June in this paper's simulation).

Their model suggests 510,000 deaths, which implies a death rate of $0.94 \%$. These two figures are similar to those produced by this paper's model. If the Imperial College figures for percentage of infections and death rate were regarded as more accurate, then applying a correction factor of $0.094 / 0.099 \times 81 / 87$ to this paper's total gives 509,000 deaths, essentially the same result.

Within the accuracy of the data, it may be concluded that the two models are predicting pretty much the same scenario. The dates quoted should be regarded as indicative only - the aim and benefit of this model is to capture the main modes of the epidemic.

\section{Possible strategies to counter the Covid-19 epidemic}

There will be additional, indirect premature deaths when hospitals are overloaded due to medical staff being unable to provide all patients with the care they need. This would add to the figures for premature death for Options 0,1 and 2. However, given the relatively short period predicted for extreme stress on the hospitals, the already very high figures would not be expected to rise significantly.

5.1 Option 0: "Business as usual", where hospitals are overwhelmed; Wuhan fatality rates of Table 2 are applied

Figure 2 gives the number of infectious people as a function of time. Like other countries, the UK does not currently have the hospital capacity to cope with the numbers of people who would need hospitalization. Hence, the fatality rates for this option are represented by Table 2, which is likely to best represent the situation where the UK's hospitals are overwhelmed by a high number of severe cases.

The trend of the epidemic shown in Figure 2 is unaltered, but the overall death rate now goes up to $1.7 \%$. The resultant number of deaths rises in proportion to 990,000. 16.7 million years of life are expected to be lost. This implies the average victim loses just under 17 years of life expectancy, significantly below the life expectancy, 42 years, that would have been lost if all age groups had been equally at risk.

The UK's Prime Minister, Boris Johnson, has likened the struggle against COVID-19 to a war. Continuing with that comparison, the UK sustained 880,000 military casualties and 2,000 civilian deaths ${ }^{22}$ in World War I. Since the soldiers, sailors and airmen killed would have been predominantly young, a reasonable estimate of the total loss of life expectancy incurred is approximately 44 million life years.

We may put this very large figure on a scale that is easier to understand by dividing it by the life expectancy from the present onwards for the average UK citizen in 2020, a unit we may name the population-average life expectancy or plex. We may take the plex for 2020 as 42 years in round figures (for comparison, life expectancy is 81 years at birth, 41 years for a forty-year-

\footnotetext{
${ }^{22}$ www.parliament.uk, The fallen. Military strength and deaths in combat (excerpt from G. Thompson et al., Olympic Britain: Social and economic change since the 1908 and 1948 London Games). London: House of Commons Library (2012) https://www.parliament.uk/business/publications/research/ olympic-britain/crime-and-defence/the-fallen/
} 
old and 9 years for an eighty-year-old). It is the amount of life a person selected at random from the population would, on average, lose if he or she were unfortunate enough to be killed tomorrow. On this basis the loss of life in WWI is equivalent to 1.05 million average UK citizens losing their lives today (i.e., 1,050,000 plex-2020).

In WWII, 384,000 UK military personnel were lost, while 70,000 in the civil population lost their lives. This implies a loss of approximately 22 million years of life expectancy, which translates into 525,000 plex-2020, as shown in Table 3.

Table 3. UK loss of life in World War I and World War II.

\begin{tabular}{ccc}
\hline & $\begin{array}{c}\text { Loss of life } \\
\text { (years) }\end{array}$ & $\begin{array}{c}\text { Loss of life } \\
\text { (plex-2020) }\end{array}$ \\
\hline WWI & $\sim 44,000,000$ & $\sim 1,050,000$ \\
WWII & $\sim 22,000,000$ & $\sim 525,000$ \\
\hline
\end{tabular}

The loss of life under Option 0 will be just under 400,000 plex-2020, about four fifths of that lost in WWII and $40 \%$ of the losses in WWI. This scale of potential loss amply justifies Boris Johnson's analogy. One difference from those wars, however, is that although the eventual level of human loss of life to COVID-19 is not known with great precision in advance (what is available is only a model prediction), the attainment of population immunity more or less guarantees eventual victory in the battle against the virus.

5.2 Option 1: 4 month lockdown followed by lifting of restrictions; start time chosen to minimize deaths and give the UK population immunity from further COVID-19 epidemics; Wuhan fatality rates (Table 2 )

In the absence of improved medical care (thousands more ventilators, improved therapies) to reduce fatality rates or of a new vaccine to give population immunity without people contracting the infection, one method of reducing fatalities is to reduce $R_{0}$ by lockdown for an interval starting at an optimally chosen date and then to remove the restrictions. By implementing lockdown Wuhan Province managed to reduce the average number of transmissions from an infectious person to 1.05 within about a week. ${ }^{18}$

Setting the interval of lockdown at 120 days, it is found using the model that the optimal policy is to institute a step reduction in $R_{0}$ on 25 May 2020, from a starting value of 2.35 down to 1.05. The latter figure is exactly that observed at Wuhan after lockdown. The step change is made 9 days before the peak observed in Option 0. Figure 3 shows the transient of infectious people, which now reaches a maximum that is about 3 million lower than under Option 0. Hospital facilities can, however, still be expected to be overwhelmed and so the Wuhan fatality rates of Table 2 have been used for this Option.

The peak number of deaths will be reduced to 656,000 , with an associated loss of 11.1 million years of life. Under the strategy outlined above, the cumulative total of people infected will be 38.7 million, just over the number, 38.5 million, required to give population immunity. This means that no second significant outbreak would occur. 


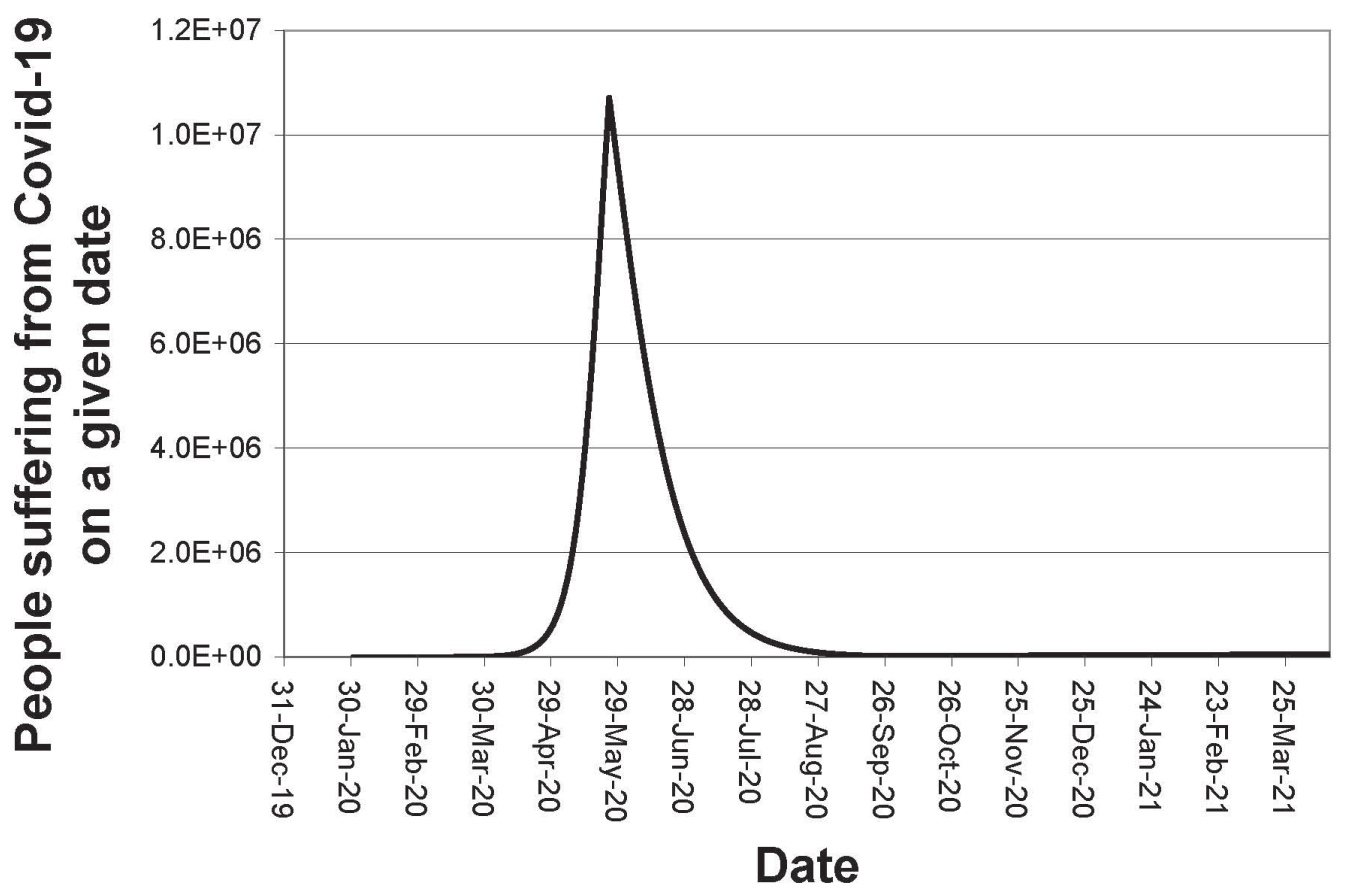

Figure 3. Number of patients suffering from COVID-19 at a given date (Option 1).

Option 1 restores about a third of the life expectancy lost in Option 0, around 5,620,000 years or 134,000 plex-2020 (Table 4).

Getting the timing right is, however, critical; implementing the lockdown too early or too late produces strongly suboptimal results; a month too early or a month too late yields a result only slightly better than business as usual. A reasonable window for action, when most of the benefit may be gained, is essentially no more than 5 days either side of the optimal figure.

5.3 Option 2: early 12 month lockdown followed by lifting of restrictions; Wuhan fatality rates (Table 2)

For a lockdown period of 12 months starting 31 March 2020, the number of infections a year later will be kept down to about 1.1 million. The number of deaths may be estimated using the nonoverwhelmed overall fatality rate of just below $1 \%$ and is therefore about 11,000 by the end of March 2021. However, this is not the end of the story as, unfortunately, lifting the restrictions after a year will allow the disease, not totally eradicated at that point, to re-establish. The result will be an epidemic that is little different from what would have occurred a year earlier. See Figure 4, which depicts an epidemic that is in all essentials a delayed version of that shown in Figure 2.

Without measures having been taken to increase hospital capacity in the interim, hospitals will be overwhelmed and the Wuhan fatality rates of Table 2 are appropriate. A relatively small number of premature deaths, 4,500, are still avoided and the corresponding number of years of life expectancy restored is 76,500 or 1,800 plex-2020 (Table 4). 


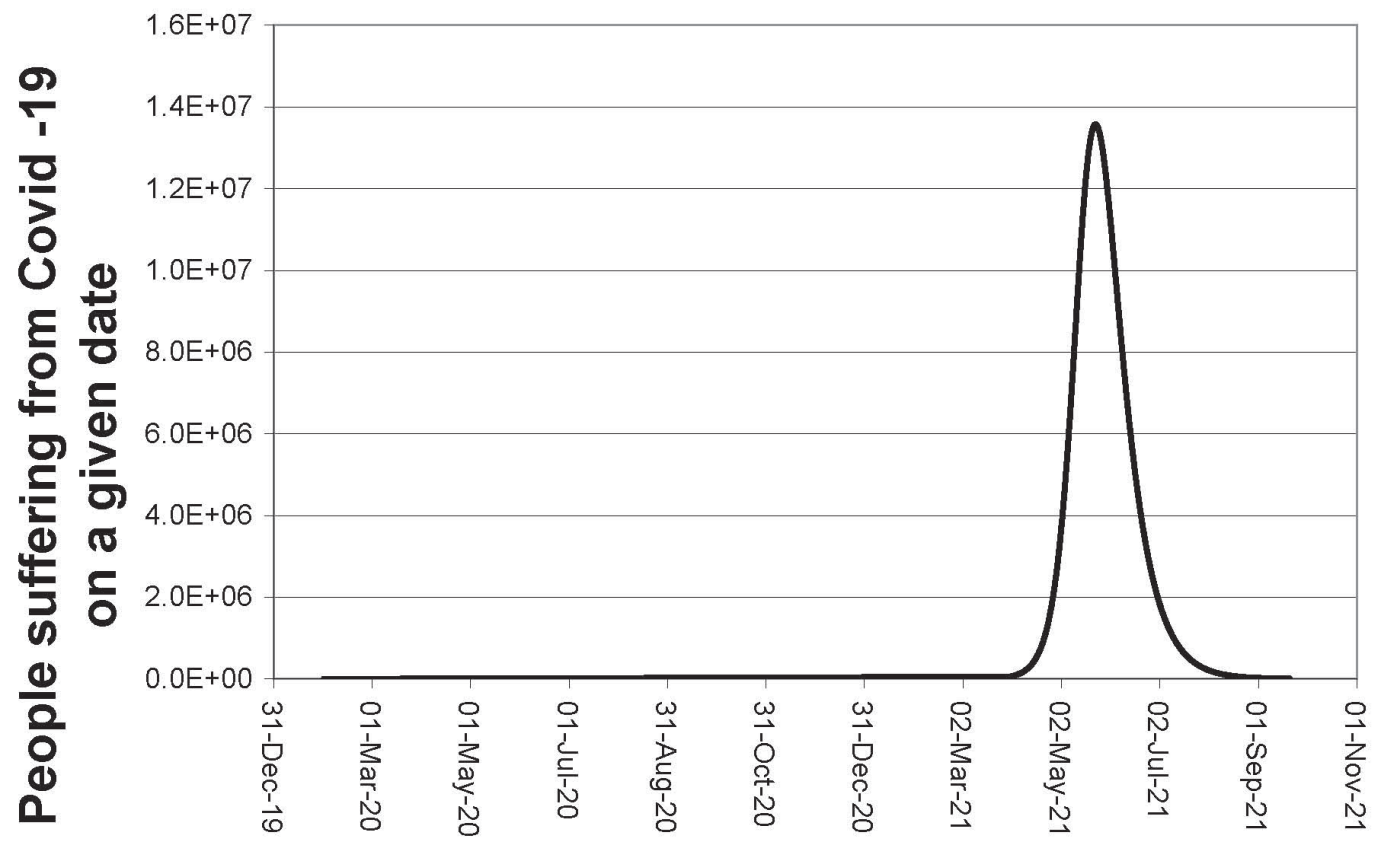

Date

Figure 4. Number of patients suffering from COVID-19 at a given date (Option 2).

The numbers remain low through the period of restriction, but the epidemic takes off about 2 months later.

5.4 Option 3: early 12 month lockdown followed by a second, optimally timed 4 month lockdown later; greatly enhanced hospital capacity made available by the end of 12 months; Table 1 fatality rates

The initial lockdown is for 1 year, starting 31 March 2020. The epidemic starts up again as shown in Figure 4, but then there is a second lockdown lasting 120 days, starting 15 May 2021 (Figure 5). The effect of the second lockdown is to reduce the final total number of infections from 58.4 million under Option 0 to 38.4 million under Option 3, approximately the level needed to induce population immunity.

The enhanced capacity assumed for the hospitals means that they are not now overloaded and so the death rates of Table 1 may be used. There are now 368,000 premature deaths, and the total loss of life expectancy is 5.4 million years. This represents a saving of 11.3 million expected years or 270,000 plex-2020 compared with Option 0.

5.5 Option 4: early 12 month lockdown with widespread vaccination at the end of the lockdown period using a newly developed vaccine; Table 1 fatality rates

It is assumed under Option 4 that 38.5 million people will have been vaccinated by the end of the year-long lockdown, a sufficient number on its own to give population immunity. This is simulated by adding 38.5 million to the model's number of infected and recovered people on 31 March 2021. The resulting transient in infectious people is shown in Figure 6. 


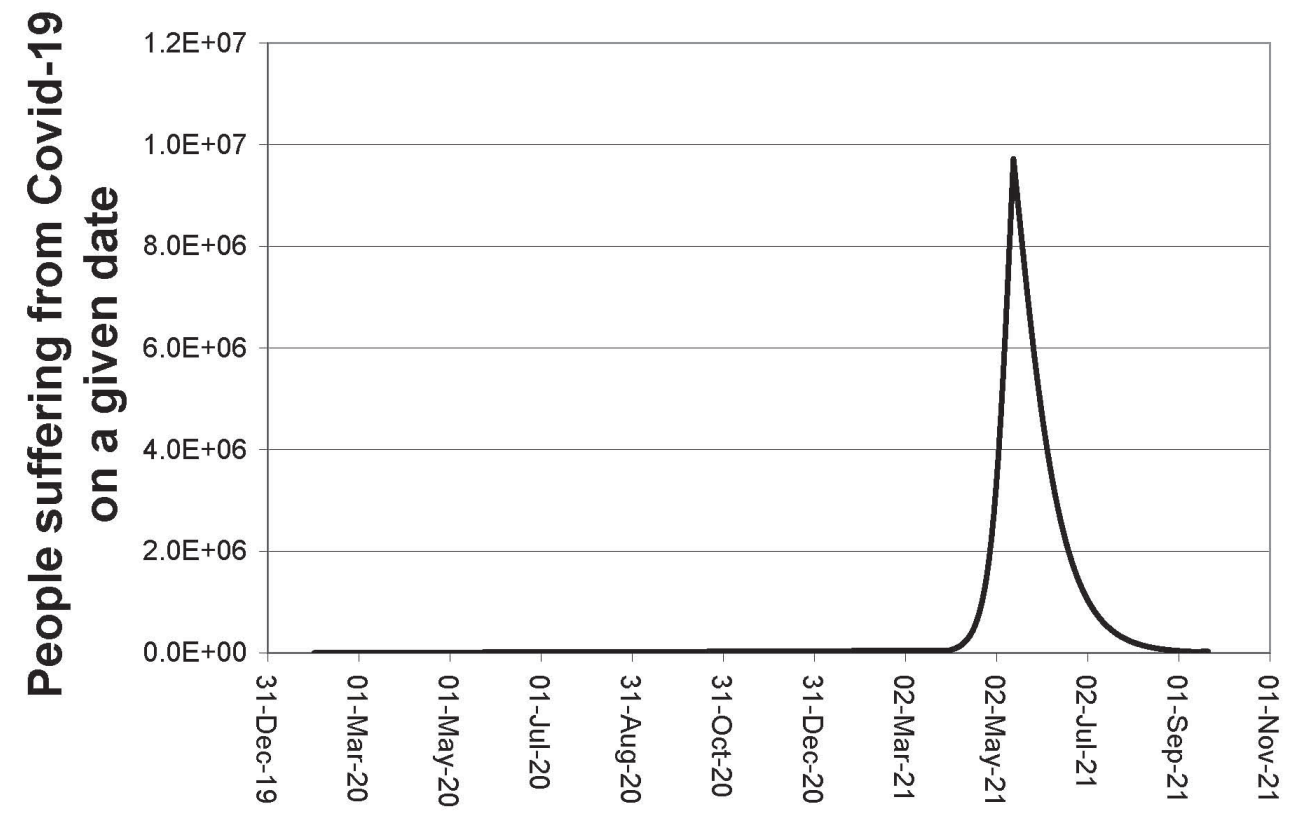

\section{Date}

Figure 5. Number of patients suffering from COVID-19 at a given date (Option 3). The figure is essentially a delayed version of Figure 2.

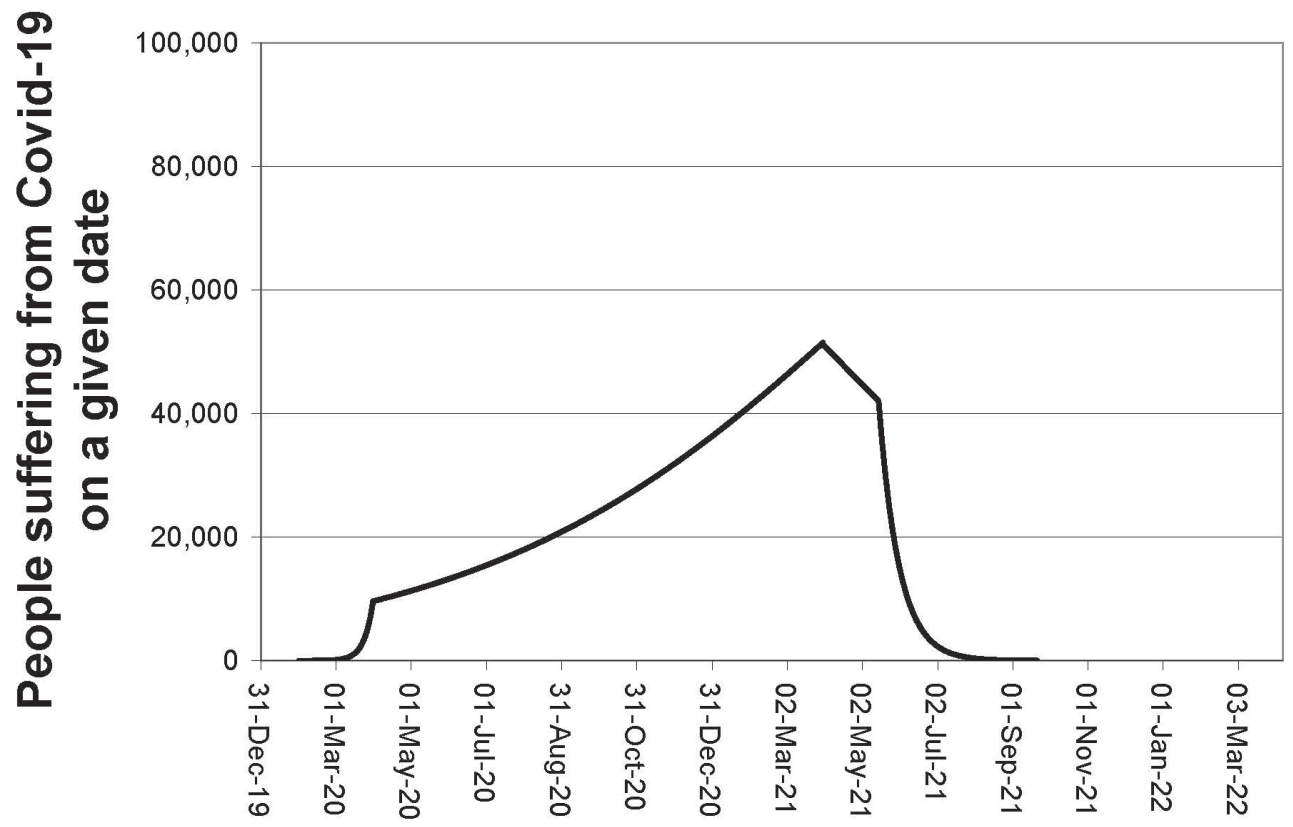

\section{Date}

Figure 6. Number of patients suffering from Covid-19 at a given date (Option 4). 
The final total number of people to have been infected will be 1.3 million. Given that hospitals should not be overwhelmed, the fatality rates listed in Table 1 are appropriate, implying a total of about 13,000 premature deaths. This is less than the average annual number, 17,000, of influenza deaths in England over the past 5 years. ${ }^{23}$ Each COVID-19 victim would lose, on average, 14.6 years of life. Hence the total loss of life expectancy is 189,000 years or 4,500 plex-2020 (Table 4). Compared with Option 0, Option 4 would avoid 977,000 premature deaths and restore 16.5 million life years or 393,000 plex-2020.

\section{J-value analysis of the options}

6.1 J-value analysis based on valuation of life years, as described in Section 2.1

Table 4 summarizes the characteristics of the 5 possible options at the UK's disposal to combat COVID-19.

Table 4. Comparison of the options against COVID-19.

\begin{tabular}{lcccccc}
\hline & $\begin{array}{c}\text { J-value } \\
\text { justified } \\
\text { cost/10 } £\end{array}$ & $\begin{array}{c}\text { Restoration } \\
\text { of life/ years }\end{array}$ & $\begin{array}{c}\text { Restoration } \\
\text { of life } \\
\text { (plex-2020) }\end{array}$ & $\begin{array}{c}\text { Residual loss } \\
\text { of life/years }\end{array}$ & $\begin{array}{c}\text { Residual } \\
\text { loss of life, } \\
\text { (plex-2020) }\end{array}$ & $\begin{array}{c}\text { Approx. } \\
\text { end date } \\
\text { of crisis }\end{array}$ \\
\hline Option 0 & 0 & 0 & 0 & $16,688,465$ & 397,344 & Sept. 2020 \\
Option 1 & 1,395 & $5,618,411$ & 133,772 & $11,070,054$ & 263,572 & Sept. 2020 \\
Option 2 & 19 & 76,493 & 1,821 & $16,611,972$ & 395,523 & Sept. 2021 \\
Option 3 & 2,817 & $11,317,153$ & 269,456 & $5,371,312$ & 127,888 & Sept. 2021 \\
Option 4 & 4,095 & $16,499,887$ & 392,854 & 188,578 & 4,490 & Sept. 2021 \\
\hline
\end{tabular}

Option 1, an optimally timed 4-month lockdown, would reduce the loss of life from 400,000 to 260,000 plex-2020, a substantial saving. Applying the J-value at the recommended point of $J=1$ gives the maximum that should be spent on the 4-month, optimally timed, lockdown as $£ 1,395,000$ million. Clearly massive spending is justified in principle for this option. However, this constitutes about two thirds of the UK's GDP of $£ 2,215,000 \mathrm{M}$ in $2019 .{ }^{24}$ In practice it would be difficult to get the timing right and a saving of 100,000 plex-2020 might be more realistic. Nevertheless such an outcome would still justify the spending of a million million pounds.

Option 2, a lockdown of 12 months followed by a return of the epidemic on a similar scale to that occurring with Option 0 , would make little difference. It would be worth spending only $£ 19,000 \mathrm{M}$ on the benefit it would produce, and it is clear that such a sum would not begin to cover the economic cost of a year's lockdown for the nation.

Option 3, a year's lockdown, allowing time for sufficient supplies of both ventilators and intensive care beds to be built up, followed by an optimally timed 4 month lockdown would reduce the loss of life to 130,000 plex-2020, i.e. about a third of that under Option 0. Applying the $\mathrm{J}$-value criterion at $J=1$, the justifiable cost of enabling this scenario to happen is $£ 2,820,000 \mathrm{M}$. This enormous sum is, however, greater than the country's GDP in 2019.

${ }^{23}$ Public Health England, Surveillance of Influenza and other Respiratory Viruses in the UK Winter 2018 to $2019 \mathrm{https}: / / w w w . g o v . u k / g o v e r n m e n t / s t a t i s t i c s / a n n u a l-f l u-r e p o r t s$

${ }^{24}$ ONS, GDP first quarterly estimate time series, Sheet A2, GDP and GVA in $£$ million (2020) https:// www.ons.gov.uk/economy/grossdomesticproductgdp/datasets/secondestimateofgdp 
In Option 4, 38.5 million people are vaccinated in the closing stages of a 12-month lockdown. This strategy would be highly successful in combating the virus, with a loss of less than 5000 plex-2020. The J-value analysis of Section 2.1 suggests that the justifiable cost of such a set of countermeasures (a year's lockdown, development of an effective vaccine and widespread immunization within a timescale of about 12 months) would be $£ 4,095,000 \mathrm{M}$. However, this sum falls not far short of twice the UK's GDP in 2019.

Clearly Option 2 is a non-starter. Options 1, 3 and 4 would each prolong a lot of human life. Option 3 relies mainly on technology that has been developed already and the challenge would be to get enough equipment manufactured in time, enough temporary hospitals built and enough trained staff to operate them. An additional bonus, not accounted for in the figures presented, could come from the repurposing of existing anti-viral therapies to attack the coronavirus, which would improve the survival rates of Table 1.

Option 4 is obviously highly attractive from the point of view of rendering the coronavirus less harmful in practice than an average year of influenza. There is also encouraging news on vaccine development, with a US vaccine being tested on humans in mid-March 2020 and a UK vaccine being applied to animals at the end of March 2020 preparatory to human testing scheduled for April. ${ }^{25}$

Very great expenditure on each one of Options 1, 3 and 4 is justified under the J-value method outlined in Section 2.1. However, it is necessary to consider the J-value GDP constraint when the justifiable amounts are comparable with a full year's GDP. This consideration is the subject of Section 6.2.

\subsection{The effect of the J-value GDP constraint for exceptionally large justifiable costs}

The UK Government (along with many other governments in the developed world) appears to be pursuing a mixture of Options 3 and 4: Option 4 ideally, but with some version of Option 3 as a fall-back position if no vaccine becomes available in time. It is also plain from the measures that have been announced by the Government that it intends to spend very large sums of money. ${ }^{\mathbf{2 6}}$ However, it is also clear that the Government's lockdown will cause cutbacks to GDP. For example, as of 19 March 2020, the hospitality (pubs, restaurants, cafés) and entertainment (cinemas, clubs, theatres) industries have been instructed to suspend activities indefinitely and the same applies to sports and leisure.

The most effective countermeasure in terms of lowering life lost is Option 4, which restores 16.5 million years of life (393,000 plex-2020) that would otherwise have been lost (with Option 0). However, if the cost borne by UK citizens of implementing Option 4 is so great that their total life expectancy is reduced by an even greater amount, then the option should not be pursued. This condition may be written:

$$
N \Delta X \geq 16,500,000
$$

where $N$ is the number in the UK population, viz. 67,000,000. Solving for $\Delta X$ gives a permissible decrease in population-average life expectancy of 0.25 years. This translates into

${ }^{25}$ Whipple, T., Vaccine trials start at Porton Down in race to find a cure. The Times (21 March 2020).

${ }^{26}$ Swinford, S., Coronavirus: Chancellor Rishi Sunak announces 'enormous' wage bailout. The Times (21 March 2020). 
the following condition on the fractional fall in GDP per head, $\Delta G / G_{0}$ :

$$
\frac{\Delta G}{G_{0}} \leq 1-\left(1-\frac{\Delta X}{X_{0}}\right)^{\frac{1}{1-\varepsilon}}
$$

Solving condition (12) when $X_{0}=41.8, \Delta X=0.25$ and $\varepsilon=0.25$ gives

$$
\frac{\Delta G}{G_{0}} \leq 6.4 \%
$$

This implies that a recession resulting in a fall in economic output greater than $6.4 \%$ per person over a prolonged period would cost more life than would be restored by Option 4.

The theory behind the calculation assumes quasi-steady conditions and it is not expected that a temporary fall of $6.4 \%$ followed by an immediate recovery would contravene equation (13). However, a prolonged recession of this magnitude would be expected to have the deleterious effect on life expectancy described.

For comparison, in the economic recession of 2007-9, UK GDP per head fell by $6 \%$ in real terms between 2007 and 2009 (see Figure 7), and did not cover to its 2007 level until 2015. The effect of the recession on life expectancy is seen in Figure 8, where the life expectancy at age 42 is displayed as an approximate proxy for population-average life expectancy. The effect of the reduction in GDP per head appears about 3 years after the severe dip of 2008-9 occurs, when the clear upward trends for both men and women are markedly reduced. Flat-lining is apparent after 2014. Moreover, a fall in life expectancy at birth is observable in the case of females born to the most deprived $10 \%$ of the population. ${ }^{27}$

While J-value analysis would predict some degree of concavity for the graphs shown in Figure 8, nevertheless it is clear that, without the economic recession of 2007-2009, life expectancies at age 42 would have been at least 3 months greater by 2017 .

The Centre for Economics and Business Research is now predicting that the coronavirus pandemic will cause global GDP to decline twice as much as during the financial crisis of 2007-9. Furthermore, it raises the prospect of a 1930s-style recession. ${ }^{28}$ Such an outcome, if it were to come about, would cause a loss of life-years to the UK population that would dwarf the predicted toll under Option 0.

Based on a sector-by-sector analysis of the effects of lockdown on the German economy, the IFO Institute of Munich has recently concluded that a partial shutdown of 2 months duration will reduce German annual GDP by between 7.2 and 11.2 percentage points, while extending the period by a further month would cause annual GDP to fall by between 10 and $20.6 \% .{ }^{29} \mathrm{Such}$ outomes, if they were transferred to the UK, would be sufficient to cause significantly greater

${ }^{27}$ Marshall, L. et al., Mortality and Life Expectancy Trends in the UK: Stalling Progress, The Health Foundation (2019).

${ }^{28}$ Centre for Economics and Business Research (22 March 2020) https://cebr.com/reports/a-worldrecession-is-now-almost-a-certainty-with-global-gdp-set-to-decline-twice-as-much-as-during-thefinancial-crisis-the-challenge-now-is-to-prevent-the-recession-from-turning-into-a-1930s-style/

${ }^{29}$ Dorn, F. et al., The Economic Costs of the Coronavirus Shutdown for Germany: a scenario calculation (EconPol Policy Brief 25) (completed 22 March 2020). Munich: IFO Institute (2020).

Nanotechnology Perceptions Vol. 16 (2020) 


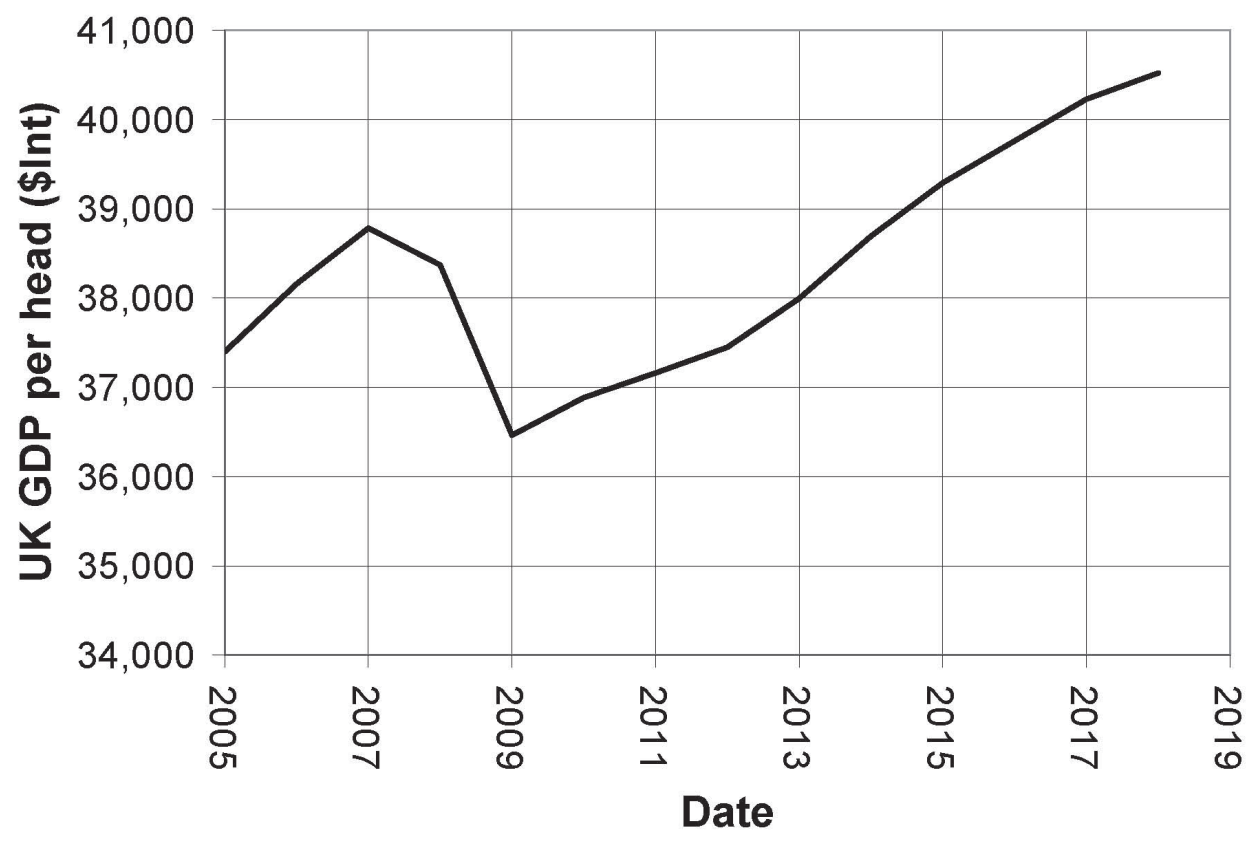

Figure 7. GDP per head in international dollars (purchasing parity preserved). Source: World Bank https://databank.worldbank.org/reports.aspx? source=2\&type=metadata\&series=NY.GDP.PCAP.PP.KD\#

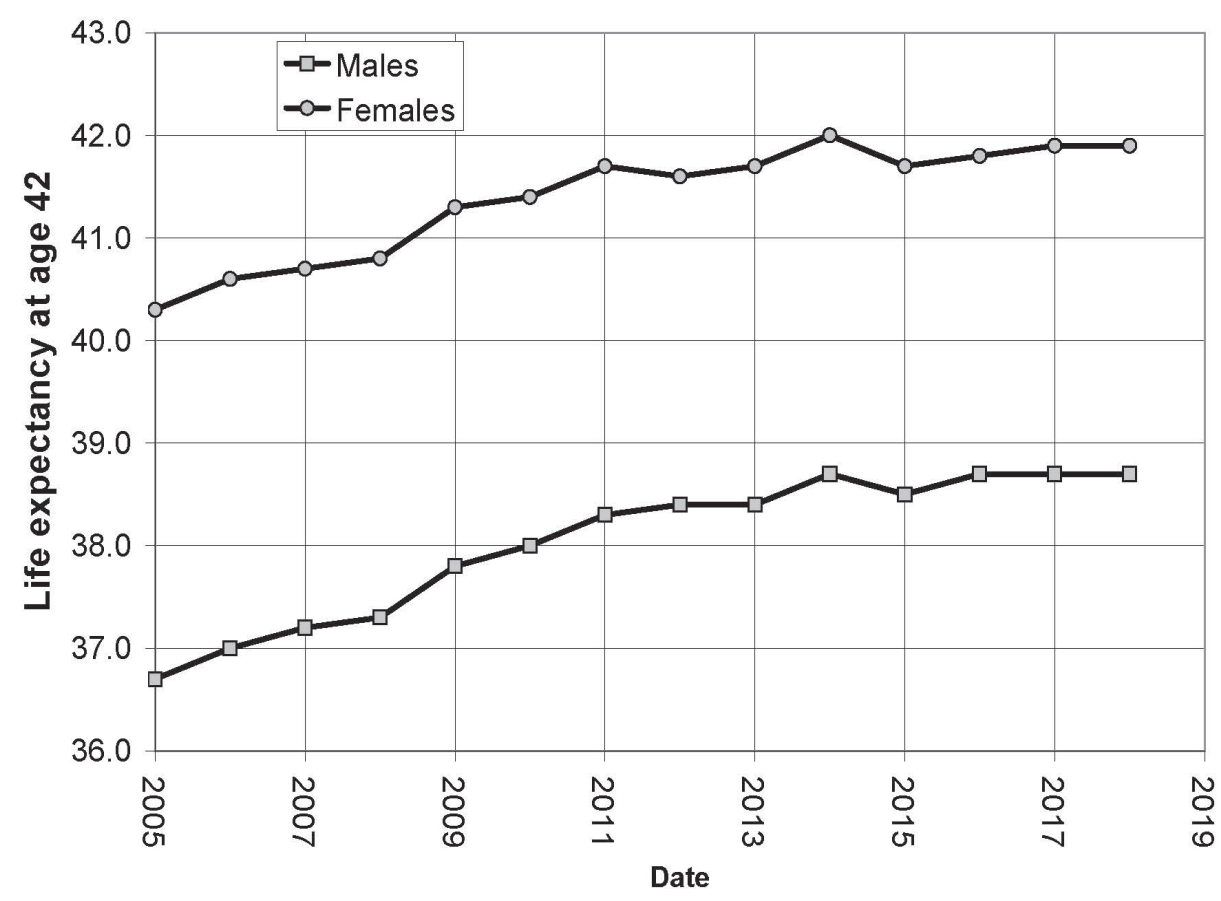

Figure 8. UK life expectancy at age 42. Source: Office for National Statistics https://www.ons.gov.uk/peoplepopulationandcommunity/birthsdeathsandmarriages/lifeexpectancies/ datasets/expectationoflifeprincipalprojectionunitedkingdom 
loss of life than would be gained by the lockdown measure, especially if the partial shutdown of the economy were to last more than 2 months in total.

Thus, the Government faces health and economic challenges of comparable severity. It needs to institute reasonable countermeasures against COVID-19 but it needs, at the same time, to attempt to restrict the coming recession to not much worse than the 2007-9 financial crash.

\section{Uncertainties in the modelling of the epidemic and its effects}

Information on the coronavirus epidemics around the world is growing and there will be scope for refinement of the numbers presented here, especially if, as looks likely, time is bought through governments adopting variants of either Option 3 or Option 4. The exact size of the outbreak and all the dates quoted from the model should be regarded as indicative only. However, knowledge about the main modes of the epidemic's behaviour and the orders of magnitude of the numbers of people affected under the various options is regarded as good enough to guide policy decisions.

\section{Conclusions}

There is no doubt that the new coronavirus infection is posing and will continue to pose a severe challenge to the UK and all countries around the world.

Less developed countries do not have such good public health systems, but on their side is the fact that their populations contain high proportions of young people who have been observed to be much less vulnerable to severe effects. By contrast, the developed countries have better health systems, but on the other hand they have much higher percentages of older people who are more likely to be badly affected.

The range of possible options has been explored using the epidemic model developed in the paper. A "business as usual" approach would lead to the epidemic being over by September 2020, but it would lead to a loss of life comparable to that suffered by the UK in the Second World War. An optimally timed 4-month shutdown starting a little before the peak would otherwise have been reached could cut casualties by a third and again the epidemic would be almost over by September 2020. However this option would still leave a high residual number of fatalities caused by the virus.

A 12-month lockdown on its own would merely delay the onset of the epidemic by a year and the number of deaths would be little altered from the business as usual approach. This strategy (Option 2 of the paper) should not be followed.

However, a 12 month lockdown during which fully-equipped hospital capacity was prepared, followed by an optimally timed 4 month shutdown could cut casualties by two thirds.

A 12 month lockdown during which a vaccine was developed and a large-scale immunization programme was carried out would cut the damage done by the coronavirus to less than the average toll on people caused by influenza each year.

Using the J-value without constraint suggests that exceptionally high spending would be justified for the three strategies able to make a significant reduction in the numbers of cases and deaths compared with the unmitigated epidemic. However, such high spending is likely to come up against the J-value GDP constraint, whereby the measure should not decrease GDP per head so much that the UK population as a whole loses more life than it gains from the countermeasure. 
The challenge for the UK Government will be to manage its interventions so that the inevitable impending recession is not significantly worse than that induced by the 2007-9 financial crash. The pandemic nature of the problem means that all governments around the world face a similar task.

\section{Appendix A. Model of the Covid-19 epidemic in the UK}

Let the number of people in the national population be $N$ and let the number of people who are infected and infectious the coronavirus SARS-CoV-2 be $n$.

A just-infected person will undergo an incubation period, $\tau_{\text {inc }}$ (about 5 days), and will then be liable to infect others for a risk period, $\tau_{\text {risk }}$ (about 10 days). It is possible, however, that the person may be infectious in the later stages of the incubation period. Allowing for this, a general statement of the average time, $\tau_{\text {inf }}$, to infect others, measured from the time the first person is infected, may be written:

$$
\tau_{\text {inf }}=r_{1} \tau_{\text {inc }}+r_{2} \tau_{\text {risk }} \quad\left\{\begin{array}{l}
0<r_{1} \leq 1.0 \\
0<r_{2} \leq 1.0
\end{array}\right.
$$

When the person is not infectious during the incubation period, $r_{1}=1.0$ and when

the infectiousness is uniformly distributed over the normal risk period, $r_{2}=0.5$.

It will now be assumed that all the transfer of virus will occur by a single contact $\tau_{\text {inf }}$ days after the person was infected. The number of people infected by the average person will be the basic reproduction number, $R_{0}$. By this model, $\tau_{\text {inf }}$ marks the beginning and end of the onward transmission of infection for the person passing on the virus. Thereafter he or she will either recover or, in a small fraction of cases, become ill and die. Clearly the instant of transmission also marks the start of the infection for the individual receiving it. Hence $\tau_{\text {inf }}$ may be regarded as the average time between generations of infected people.

Infections will be passed continually from one person to another, and we will cater for this by assuming that at any instant the periods since infection amongst those with the possibility to infect will have a uniform distribution between 0 and $\tau_{\text {inf }}$ days. Let us divide the average generation time, $\tau_{\text {inf }}$, into a large number, $M$, of time intervals, $\delta t$, where

$$
\delta t=\frac{\tau_{\text {inf }}}{M}
$$

Let us also divide the $n$ people with the potential to infect others, whom we may regard as the current generation of infected people, into $M$ groups, each of which contains $n_{G}$ people whose infection times are within $\delta t$ of each other, where

$$
n_{G}=\frac{n}{M}
$$

Making $M$ very large implies $\delta t \rightarrow 0$ from equation (A.2), and this allows us to consider that all the people in each group were infected at the same time. Although this will only be an 
approximation early on, there will no problem in practice in regarding $M$ as very large fairly soon for an epidemic predicted to reach many millions of people.

Let us assign the name Group 1 to the group in the current generation that contains the people who were infected first and are about to pass on their infection. Group 2 will contain those with the second oldest infections, and so will pass the disease on next. We may then continue this process of assignment until we encounter the last group of the current generation to have been infected, which will be given the name Group M.

Figure 9 shows how the $M$ groups of the current generation pass on their virus load to the next generation. The figure illustrates an important fact about an epidemic, namely that the number of people to whom the average infected person will pass on an infection will fall over time as the people in the contact group are increasingly likely either to be infected already or are immune as a result of having been a sufferer previously and having now recovered. The effective basic reproduction number will therefore be

$$
R=R_{0} p_{s}
$$

where $p_{s}$ is the probability of the person contacted by the infectious person being susceptible to catching the disease. Noting that many contacts in the real world are random, this may be taken as the fraction of susceptible people remaining in the population:

$$
p_{s}=\frac{n_{s}}{N}
$$

where the number of susceptible people, $n_{s}$, is given by:

$$
n_{s}=N-n-n_{r}
$$

Here $n_{r}$ is the number of people who have been infected and have now recovered, or, in a small number of cases, died.

By assumption, the infected people of the current generation will cease to be infectious immediately after the point when they have the chance to transfer their infection onto others (which will not always happen, as we have seen, due to the growing shortage of susceptible people). This will lead to an increase, $\delta n_{r}$, in the number of previously infectious people. At the instant of possible transfer, this will be the number in the group, $n_{G}$ :

$$
\delta n_{r}=n_{G} .
$$

Combining equations (A.2), (A.3) and (A.7) gives:

$$
\frac{\delta n_{r}}{\delta t}=\frac{n}{\tau_{r \text { inf }}}
$$

As $\delta t \rightarrow 0$ as a result of making $M$ very large, this may be written in differential form:

$$
\frac{\mathrm{d} n_{r}}{\mathrm{~d} t}=\frac{n}{\tau_{\mathrm{inf}}} .
$$

Solving this differential equation from $n_{r}(0)=0$ gives the number of people who were infectious in the past and who have now either recovered or died.

There will be an increase, $\delta n_{1}$, in the number of people infected immediately following the infection event, which may be found by multiplying by the currently effective reproduction number, $R$ (see note under Figure 9):

$$
\delta n_{1}=R n_{G} .
$$




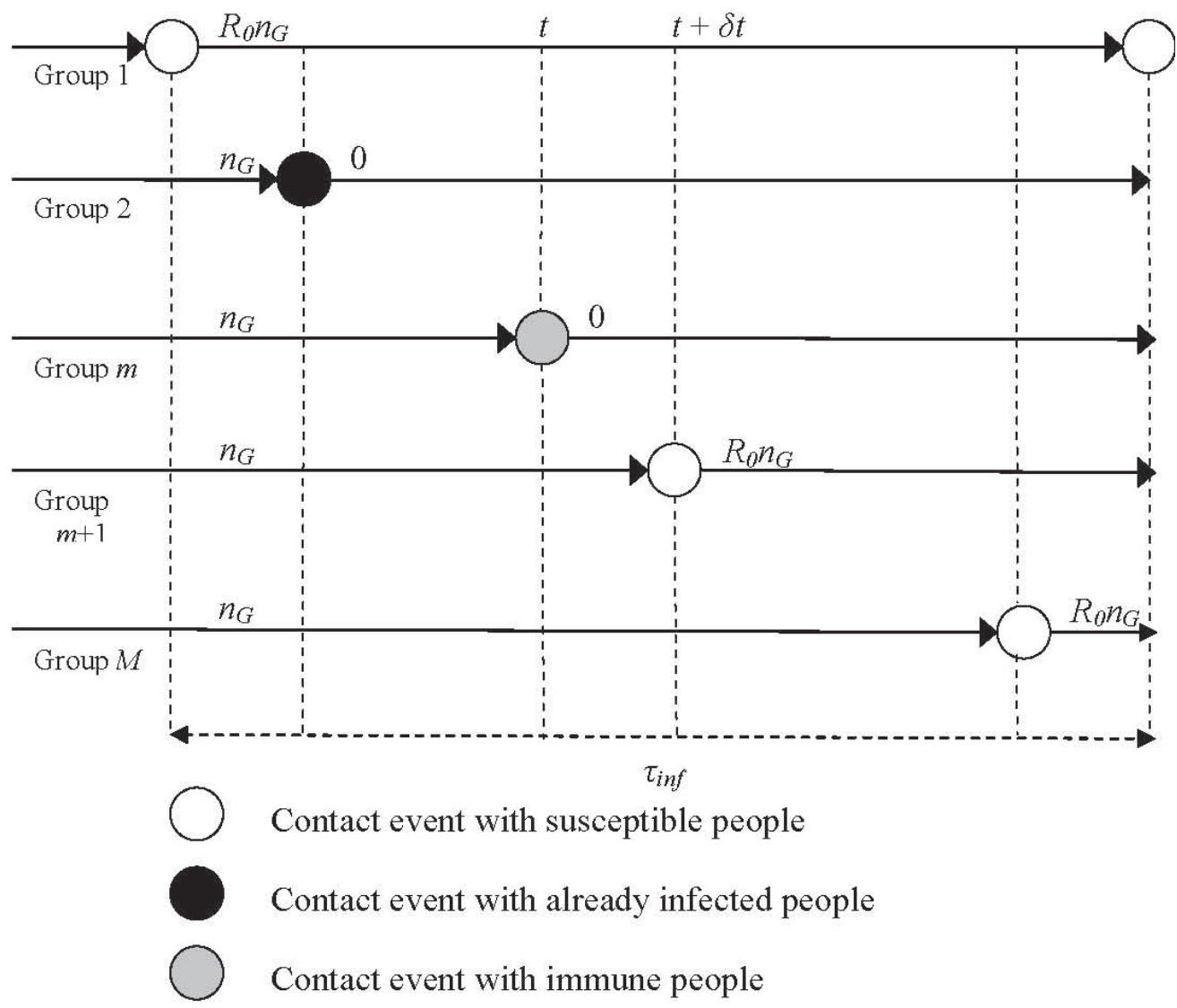

Figure 9. Schematic diagram of the transmission of infection.

A contact event with a group of susceptible people will lead to $R_{0}$ new infections, but no new infections will arise from a contact event with a group of people who are already infected or are immune. The total number of infected people in the next generation will be $n_{\text {Gnext }}=\left(R_{0} n_{G}+0+\ldots+0+R_{0} n_{G}+\ldots+R_{0} n_{G}\right) / M=R_{0} n_{G} n_{s} / N=R n_{G}$, where $R$ is the currently effective reproduction number, equal to the original number, $R_{0}$, multiplied by the fraction of currently susceptible people in the population $R=R_{0} n_{s} / N$.

Combining equations (A.2), (A.3), (A.4), (A.5) and (A.10) gives:

$$
\frac{\delta n_{1}}{\delta t}=R_{0} \frac{n}{\tau_{\text {inf }}} \frac{n_{s}}{N}
$$

or, as $M$ becomes large and $\delta t \rightarrow 0$,

$$
\frac{\mathrm{d} n_{1}}{\mathrm{~d} t}=R_{0} \frac{n}{\tau_{\mathrm{inf}}} \frac{n_{s}}{N}
$$

The net rate of change in the number of infectious people will be the increase in the number minus the decrease caused by people recovering or, in a small fraction of cases, dying: 


$$
\frac{\mathrm{d} n}{\mathrm{~d} t}=\frac{\mathrm{d} n_{1}}{\mathrm{~d} t}-\frac{\mathrm{d} n_{r}}{\mathrm{~d} t}=R_{0} \frac{n}{\tau_{\text {inf }}} \frac{n_{s}}{N}-\frac{n}{\tau_{\text {inf }}} .
$$

Hence

$$
\frac{\mathrm{d} n}{\mathrm{~d} t}=\frac{n}{\tau_{\mathrm{inf}}}\left(R_{0} \frac{n_{s}}{N}-1\right) .
$$

It may be observed immediately from equation (A.14) that the number of infected people will cease to increase when the fraction of susceptible people in the population has decreased to

$$
\frac{n_{s}}{N}=\frac{1}{R_{0}} .
$$

Equivalently, the fraction of the population to have contracted the coronavirus will be, from equation (A.6):

$$
\frac{n+n_{r}}{N}=1-\frac{1}{R_{0}}=\frac{R_{0}-1}{R_{0}} .
$$

This is the fraction needed to give population ("herd") immunity. Any new infection introduced to a population with population immunity will be unable to sustain itself and will die away. This does not mean that there will be no transmission, but it means that there will be fewer in each succeeding generation of infections. For an established epidemic, conditions (A.15) and (A.16) characterize the peak, when the number of people suffering from the disease is a maximum. The number will then decrease over time to zero (although susceptible people will be infected, in declining numbers, on the way down).

\section{Appendix B. Calculating the final number of deaths in each age group}

The cumulative total of infections, $n_{T o t}$, after no more are transmitted, will be the sum:

$$
n_{\text {Tot }}=n\left(t_{\text {end }}\right)+n_{r}\left(t_{\text {end }}\right)=n_{r}(\infty)
$$

where $t_{\text {end }}$ is the time when the epidemic can be declared over.

It is assumed that a third of the infections occur in the household, a third in schools and places of work and a third in the community. ${ }^{19}$ Two further assumption are made: firstly, it is assumed that a schoolchild will be twice as likely to become infected as a person working and secondly, it is assumed that infection of people over the age of 85 will occur predominantly in the home.

The number of infections, $n_{\text {Tot, a,g }}$, at age $a$ and gender $g$ for infants aged 0 to 4 and older people aged 67 to 84 will come from two sources, the home and in the community, where they will bear their share at each age and gender up to age 84 . Hence for these age groups:

$$
n_{T o t, a, g}=p_{a, g} \frac{n_{T o t}}{3}+\frac{p_{a, g}}{\sum_{g} \sum_{a=0}^{84} p_{a, g}} \frac{n_{T o t}}{3} \quad \text { for }\left\{\begin{array}{l}
0 \leq a \leq 4 \\
67 \leq a \leq 84
\end{array}\right.
$$


where $p_{a, g}$ is the fraction of people of age $a$ and gender $g$ in the country (calculated from life tables).

The number of schoolchildren will be $N \sum_{g} \sum_{a=5}^{18} p_{a, g}$ while the number of people working will be $N \sum_{g} \sum_{a=19}^{66} p_{a, g}$. Each schoolchild will get two shares in the number of infections to one share for each of the people working, which implies that the number of shares in the infections transmitted in school and at work will be $N\left(2 \sum_{g} \sum_{a=5}^{18} p_{a, g}+\sum_{g} \sum_{a=19}^{66} p_{a, g}\right)$. Hence those aged 5 to 18 will receive a fraction of the infections equal to $2 \sum_{g} \sum_{a=5}^{18} p_{a, g} /\left(2 \sum_{g} \sum_{a=5}^{18} p_{a, g}+\sum_{g} \sum_{a=19}^{66} p_{a, g}\right)$. Those aged 19 to 66 will receive the complementary fraction.

Schoolchildren will face infections at home, at school and in the community, and so $n_{\text {Tot }, a, g}=p_{a, g} \frac{n_{\text {Tot }}}{3}+\frac{p_{a, g}}{\sum_{g} \sum_{a=5}^{18} p_{a, g}} \frac{2 \sum_{g} \sum_{a=5}^{18} p_{a, g}}{2 \sum_{g} \sum_{a=5}^{18} p_{a, g}+\sum_{g} \sum_{a=19}^{66} p_{a, g}} \frac{n_{\text {Tot }}}{3}+\frac{p_{a, g}}{\sum_{g} \sum_{a=0}^{84} p_{a, g}} \frac{n_{\text {Tot }}}{3}$ for $5 \leq a \leq 18$

Similarly workers will face infections at home, at work and in the community:

$n_{\text {Tot }, a, g}=p_{a, g} \frac{n_{\text {Tot }}}{3}+\frac{p_{a, g}}{\sum_{g} \sum_{a=19}^{66} p_{a, g}} \frac{\sum_{g} \sum_{a=19}^{66} p_{a, g}}{2 \sum_{g} \sum_{a=5}^{18} p_{a, g}+\sum_{g} \sum_{a=19}^{66} p_{a, g}} \frac{n_{T o t}}{3}+\frac{p_{a, g}}{\sum_{g} \sum_{a=0}^{84} p_{a, g}} \frac{n_{\text {Tot }}}{3}$ for $19 \leq a \leq 66$

Those aged 85 or above will face only home infections, and so:

$$
n_{T o t, a, g}=p_{a, g} \frac{n_{T o t}}{3} \quad \text { for } 85 \leq a \leq 100
$$

The cumulative number of fatalities at each age and for each gender, $n_{f, g, a}$, may then be calculated as:

$$
n_{f, a, g}=f_{a} n_{T o t, a, g}
$$

where $f_{a}, a=1,2, \ldots, 100$, is the age-specific fatality rate (Table 1 or Table 2 ).

The expected total number of years of life lost, $n_{y}$, is then

$$
n_{y}=\sum_{a=0}^{100} n_{f, a, g} X_{a, g}
$$

where $X_{a, g}$ is the life expectancy at age, $a$, and gender, $g$.

Nanotechnology Perceptions Vol. 16 (2020) 
The total number of fatalities expected to be caused by the epidemic may be calculated as:

$$
n_{f}=\sum_{g} \sum_{a=0}^{100} n_{f, a, g}
$$

The life expectancy lost by the average victim who dies is then $n_{y} / n_{f}$.

\section{Appendix C. Using the J-value to find the value of a life year}

The up-front equivalent, $\delta V$, of the annual payments, $\delta G$, may be calculated by amortizing over the population-average life expectancy, $X$, using the social interest rate, $r^{*}$ :

$$
\delta V=\int_{0}^{X} e^{-r^{*} t} \delta G \mathrm{~d} t=\delta G \frac{1-e^{-r^{*} X}}{r^{*}} .
$$

Under strong intergenerational equity, the population-average life expectancy is set to the average life expectancy, $X_{1}$, of those alive at the installation of the protective measure. This implies that the charge, $\delta G$, will be borne each year by those living at the time of installation, but all succeeding cohorts will be spared any part of the financial burden.

Combining equation (6) from the main text with equation (C.1) and putting $X=X_{1}$ gives:

$$
\delta V=\frac{1-e^{-r^{*} X_{1}}}{r^{*}} \frac{1}{1-\varepsilon} \frac{\delta X}{X_{1}} G
$$

Putting $\delta X=1$ gives the value of a life year as:

$$
\delta V^{(1)}=\frac{1-e^{-r^{*} X_{1}}}{r^{*}} \frac{1}{1-\varepsilon} \frac{1}{X_{1}} G
$$

where the bracketed superscript in $\delta V^{(1)}$ implies that this is for a single life year.

The value of risk-aversion for developed countries is found ${ }^{10}$ to be $\varepsilon=0.91$. The social discount rate, $r^{*}$, should be taken as the rate of growth of GDP per head, ${ }^{10}$ found to be $2.03 \%$ per annum for the UK for the period 1961 to $2013 .^{30}$ The UK GDP per head ${ }^{31}$ in 2019 is $G=£ 33,141$. From the latest ONS life tables (for 2016-8), the population-average life expectancy for the current generation may be calculated as $X_{1}=41.8$ years. ${ }^{32}$

\footnotetext{
${ }^{30}$ World Bank, World Development Indicators: GDP per capita growth (annual\%), updated 1 February 2017. Available at http://data.worldbank.org/indicator/NY.GDP.PCAP.KD.ZG

${ }^{31}$ Office of National Statistics, Gross Domestic Product (Average) Per Head, at Current Market Prices: $S A$ [seasonally adjusted] (2020) https://www.ons.gov.uk/economy/grossdomesticproductgdp/ timeseries/ihxt/pn2

${ }^{32}$ Office of National Statistics, National Life Lables, UK: 2016 to 2018 https://www.ons.gov.uk/ peoplepopulationandcommunity/birthsdeathsandmarriages/lifeexpectancies/bulletins/ nationallifetablesunitedkingdom/2016to2018
} 